\title{
Sexual evolution
}

\author{
Dr Richard Casey, Editor-in-chief, Journal of Sexual \& Reproductive Medicine
}

$\mathrm{T}^{\mathrm{h}}$ he first issue of the Journal of Sexual $\mathcal{E}$ Reproductive Medicine marks another step toward recognizing sexual medicine as a distinct specialty. Before 1980, sexual difficulties were viewed as being largely psychogenic in nature and difficult to treat with any degree of success, and, thus, were left to the psychologists, counsellors and therapists to unravel. The relationship between sex and fertility was not well appreciated, and fertility options were few.

The introduction of intracorporal injection therapy, the first nonsurgical option with any degree of efficacy for patients with erectile dysfunction (ED), produced a burst of interest from the urological community. Physicians with predominately surgical training were being asked to treat dysfunctional couples. The etiology of ED, once considered to be largely nonorganic, was now associated with athersclerotic vessel disease, diabetes or surgical trauma. Injectables, initially only papaverine, could produce a $60 \mathrm{~min}$ erection that was suitable for prolonged activity - usually sexual activity. A significant percentage ( $5 \%$ to $10 \%$ ) of early patients developed prolonged erections that were suitable for a visit to the local emergency room for aspiration and irrigation!

The recent introduction of effective oral therapies for ED has not only revolutionized the specialty of sexual medicine but confirmed the mind-body connection. Without sexual arousal, sildenafil citrate (Viagra, Pfizer Canada Inc, Canada) does not work. Initially the domain of the urologist, noninvasive therapies for ED now allow all physicians to participate in a patient's quest for a functional erection. Without proper training, primary care physicians, just as the last class of urologists, will learn about sexual medicine 'on the job'.
The simplistic approach to treating male sexual dysfunction by treating only the erection has resulted in helpful lessons for the emerging field of female sexual dysfunction. Results are better when clinicians consider more than just the penis and include a patient's partner. This development has encouraged clinicians and scientists to examine female sexual function in more detail. In the present issue of the Journal of Sexual \& Reproductive Medicine, Basson et al (pages 11-20) closely examine sexual changes that are present in diabetic women. Sexual clinicians who treat couples will not be surprised by the complexity of sexual issues in the female diabetic. Our experience with female sexual dysfunction suggests that we take a closer look at our 'penocentric' approach to men.

While sexual response remains the dominant clinical issue that is addressed by physicians, sexual medicine encompasses the exciting area of sexuality. This includes hormone replacement therapy for both genders, transgender issues, the effects of chronic disease and mental illness, and the implications associated with assisted reproductive technology. Physicians' efforts to improve sexual function and simulate a more natural response time will drive research to examine novel drug delivery systems and devices.

Similar to other areas of science, our knowledge of sexual function has increased faster than our understanding of the topic. Technology can produce an erection on demand; pharmacology can enhance our sexual response; and sexual educators, counsellors and therapists can assist physicians in coordinating treatments properly. We will encourage dialogue across all specialties as the specialty of sexual medicine develops. 